\title{
Mitteilungen der Deutschen Schlaganfall-Gesellschaft
}

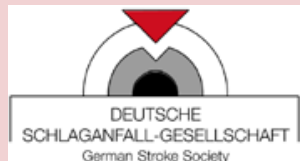

Nervenarzt 2010 - 81: 776-776

DOI 10.1007/s00115-010-3058-9

C) Springer Medizin Verlag 2010

German Stroke Society

Redaktion

Prof. Dr. med. Otto Busse

busse@dsg-berlin.org

\section{Zertifizierung für Tele-Stroke-Units}

\author{
Flächendeckende Stroke-Unit-Qualität oder \\ Akzeptanz einer Feigenblatt-Medizin
}

Die Deutsche Schlaganfall-Gesellschaft und die Stiftung Deutsche Schlaganfall-Hilfe haben unter Mitwirkung von telemedizinisch tätigen Neurologen ein Konzept für die Zertifizierung von Tele-Stroke-Units vorgelegt. Damit wird auf eine kontrovers diskutierte Entwicklung der letzten Jahre eingegangen und diese mit dem Qualitätsanspruch der Fachgesellschaft gestaltet.

Hintergrund für die Entstehung von Tele-Stroke-Netzwerken ist das bestehende Versorgungsgefälle in der Schlaganfallversorgung zwischen Regionen mit spezialisierten neurologischen Stroke Units und Regionen ohne entsprechende Einrichtungen, insbesondere in ländlichen Gegenden. Wegen der guten Erfassbarkeit der Schlaganfallsymptome in der audiovisuellen Übertragung (Videokonferenz) und der digitalen Übertragbarkeit der Schnittbildgebung liegt - gerade auch wegen des hohen Zeitdrucks in der Akutphase - ein Einsatz moderner Informationstechnologie in der Schlaganfallbehandlung nahe. In den letzten Jahren wurden daher viele strukturell sehr unterschiedliche „Telestroke“-Netzwerke aufgebaut. Kritisch wurden insbesondere Netzwerke beurteilt, die ohne konsequente Implementierung der nach internationalen Leitlinien empfohlenen Behandlungsstrukturen vor Ort eine Quali- tätsverbesserung durch die Telemedizin allein proklamierten.

\section{Strukturvoraussetzungen für die Zertifizierung von Tele-Stroke-Units}

Die Deutsche Schlaganfall-Gesellschaft hat daher mit den Initiatoren mehrerer Tele-StrokeNetzwerke Kriterien für die Zertifizierung von Tele-Stroke-Units erarbeitet. Die inhaltliche Ausgestaltung orientiert sich hierbei an der Struktur der Netzwerke, für die in wissenschaftlichen Evaluationen eine Effektivität bezüglich der Qualität der Versorgung und der Behandlungsergebnisse gezeigt wurde. Die Tele-Stroke-Units sollen nach dem deutschen Stroke-Unit-Konzept der DSG/DGN funktionieren. Damit soll die Behandlung der Schlaganfallpatienten auf einer spezialisierten und räumlich eindeutig deklarierten Krankenhausstation eines Versorgungskrankenhauses stattfinden. Die Behandlung muss durch ein multidisziplinäres Team mit in der Schlaganfallbehandlung trainierten Ärzten, Pflegekräften, Physio-, bzw. Ergotherapeuten, Logopäden sowie Sozialarbeitern geleistet werden. Die Behandlungsvorteile sollen nachweisbar durch eine hohe, kontinuierlich überprüfte Behandlungskompetenz und die strukturierte Zusammenarbeit der verschiedenen Berufs- disziplinen zu erreichen sein. $\mathrm{Pa}$ tienten mit einem akuten Schlaganfall sollen ebenso wie in Stroke Units mit Hilfe des Monitorings der Vitalfunktionen und ihrer neurologischen Defizite überwacht werden. Spezifische Therapien, wie die systemische Lyse, müssen in der Struktur der TeleStroke-Unit möglich sein, Komplikationen sollen vermieden, gegebenenfalls frühzeitig erkannt und behandelt werden. Wie in jeder Stroke Unit ist eine fortlaufende Qualitätssicherung nachzuweisen.

In dem zu überprüfenden Tele-Stroke-Unit-Konzept muss der neurologische Sachverstand als unabdingbare Voraussetzung für eine differenzierte Therapie des Schlaganfalls durch die moderne Kommunikationstechnologie sichergestellt sein, d.h. die neurologische Patientenvorstellung in der Akutphase erfolgt in der Regel über Videokonferenz. Eine kontinuierliche, qualitativ hochwertige Behandlung nach der stationären Aufnahme muss über einen neurologischen Präsenzdienst (über Festanstellung bzw. Konsiliardienst) erfolgen. Bei akutem Handlungsbedarf (z.B. im Falle sekundärer klinischer Verschlechterungen) soll außerhalb der Regeldienstzeit auch eine telemedizinische Wiedervorstellung der Patienten erfolgen - dabei sind Indikationen zur telemedizinischen Vorstellung verlässlich einzuhalten. Bei dem jeweiligen Audit ist auch zu überprüfen, inwieweit Tele-Stroke-Units in ein Netzwerk mit weiteren Tele-Stroke-Units an- derer Kooperationskliniken und einem oder mehreren Beratungszentren an überregionalen Stroke Units eingebunden sind. Neben der fachärztlichen neurologischen Beratung ist ein spezielles Qualitätsmanagement mit kontinuierlicher Schulung der beteiligten Berufsgruppen in den Kooperationskliniken, die Implementierung konsentierter Behandlungsstandards sowie die kontinuierliche Überprüfung der Struktur- und Prozessqualität als Teil der Netzwerkstruktur nachzuweisen.

Für die telemedizinische $\mathrm{Be}$ ratung sind spezielle DatenschutzVorgaben zu berücksichtigen.

Die Zertifizierung von Telestroke-Units soll - ggf. auch nur vorübergehend bis bessere Versorgungsstrukturen vorhanden sind - dabei helfen, die Behandlungsqualität auch in Regionen mit derzeit nicht ausreichend vorhandenen neurologischen Spezialeinrichtungen zu sichern. Zudem soll sie gleichzeitig einem Missbrauch der Telemedizin als „Ersatz neurologischer Stroke Units“ vorbeugen.

\section{Verfasser}

\section{Prof. Dr. Heinrich Audebert}

Ärztliche Leitung Neurologie

Campus Benjamin Franklin

Charité Universitätsmedizin Berlin

Tel.: +49 3084452297

Fax: +493084454264

\section{Prof. Dr. Otto Busse}

Deutsche Schlaganfall-Gesellschaft Reinhardtstr.14

10117 Berlin 